(C2008 IEEE. Personal use of this material is permitted. However, permission to reprint/republish this material for advertising or promotional purposes or for creating new collective works for resale or redistribution to servers or lists, or to reuse any copyrighted component of this work in other works must be obtained from the IEEE 


\title{
Content Adaptive Network Aware Joint Optimization of Wireless Video Transmission
}

\author{
Maria G. Martini and Matteo Mazzotti, CNIT/University of Bologna \\ Catherine Lamy-Bergot, THALES Communications \\ Jyrki Huusko, VTT Technical Research Centre of Finland \\ Peter Amon, Siemens AG
}

\begin{abstract}
A content adaptive, network aware approach for joint optimization of wireless video transmission is presented in this article. The joint system optimization approach has been developed in the framework of the IST PHOENIX project (www.ist-phoenix.org).

After a description of the considered crosslayer approach and of the information to be exchanged among the system component blocks, the concept of "JSCC/D controllers" is introduced. Results obtained through the demonstrator realized as proof-of-concept for the described paradigm are then shown, confirming the validity of the described joint optimization approach.
\end{abstract}

\section{INTRODUCTION}

The evolution of wireless communications is toward a more integrated and global system, meeting the requirements of both users and the industrial world, by offering convergence of different technologies and making efficient use of the existing and emerging technologies. In order to meet this goal, an efficient and flexible allocation of resources is necessary, based on co-operative optimization of the communication system components in the different layers.

Our approach, following the already known joint source channel coding and decoding (JSCC/D) approach [1], aims at developing strategies where the compression, protection, and transmission parameters are jointly determined to yield the best end-to-end system performance. Systems involving transmission of video sources over rate constrained channels actually violate the conditions upon which the optimality of the so called "separation theorem" derived from Shannon's theory [2] relies, and performance improvements may be achieved by moving from separate design and operation of source and channel codes to joint source-channel coding (JSCC) $[1,3]$.
JSCC/D techniques that include a co-ordination between source and channel encoders have been investigated, for example for transmission of audio data [4], images [5], and video [6, 7]. Some of these works adapt the transmission to the source characteristics (unequal error protection, UEP), either at the channel coding level or through source adaptive modulation (see e.g., [4]). JSCC/D techniques may also require the use of rate/distortion curves or models of the source in order to perform the optimal compromise between source compression and channel protection [5].

This joint approach requires the transmission of side information about the source and the channel through the network and protocol layers, and a realistic performance evaluation of such techniques should take this into account, although this aspect is in general neglected in the literature and few works show an effort into finding solutions for providing efficient inter-layer signaling mechanisms for JSCC/D, apart from examples such as [8].

In this article we propose a quality driven, network aware approach for wireless video transmission relying on the joint source and channel coding paradigm. Content adaptation is thus realistic here, since the transmission overhead for the exploitation of the available information about the source (e.g., in terms of motion characteristics of the video sequence and of sensitivity to channel errors of the video bitstream) is taken into account. The management of the information to be exchanged is addressed and two logical units responsible for the system optimization, in the following referred to as "JSCC/D controllers," are proposed. The demonstrator of the described system realized in the framework of the IST PHOENIX project is also presented and simulation results are given.

\section{SYSTEM ARCHITECTURE}

Figure 1 illustrates the overall system architecture developed in the framework of the 


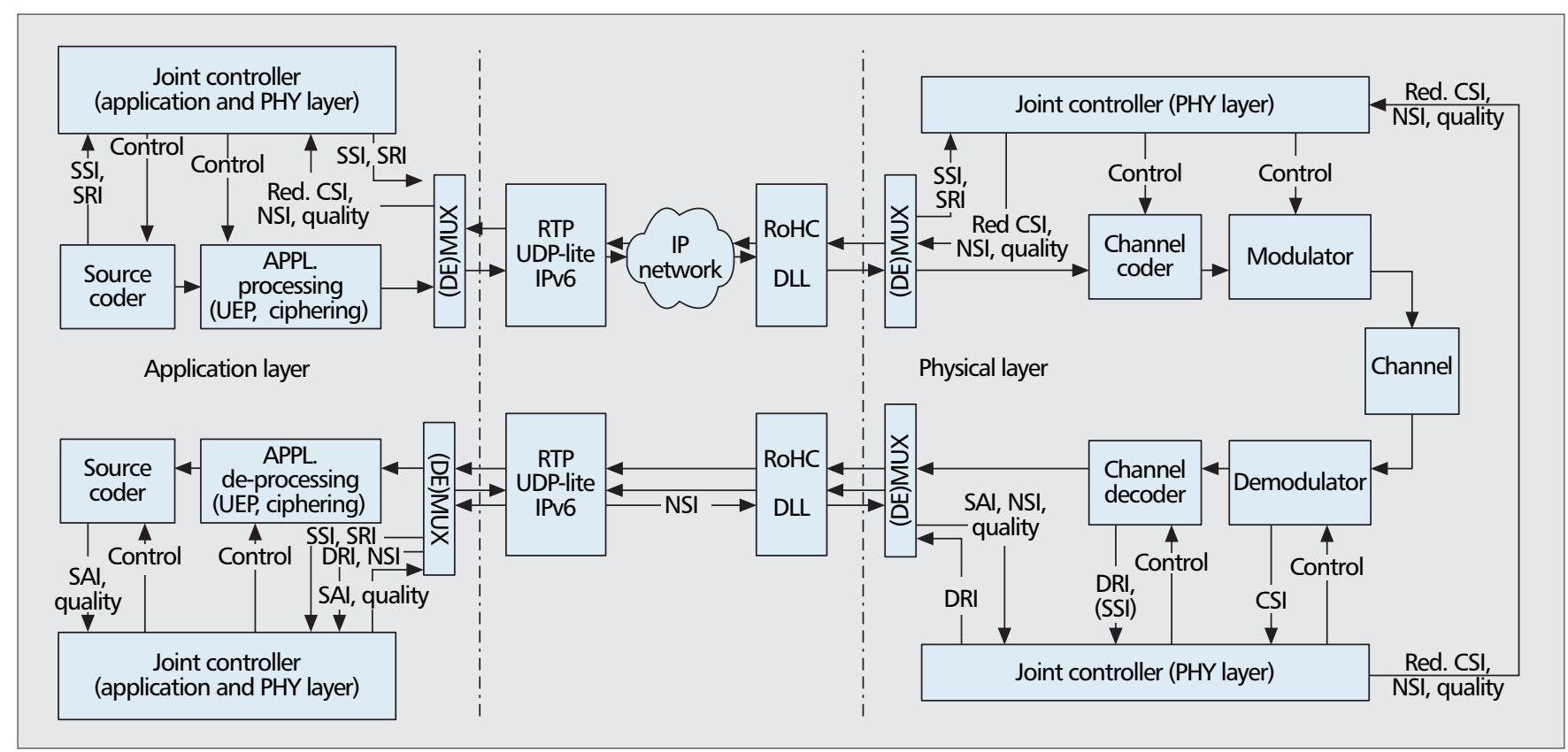

Figure 1. PHOENIX system architecture for joint optimization.

PHOENIX project, from the transmitter side in the upper part of the figure to the receiver side in the lower part, including the signalization used for transmitting the JSCC/D control information in the system. Besides the traditional tasks performed at the application level, at the network level, medium access and radio access, the architecture includes two controller units at the physical and application layers. Those controllers are used for supervising the different (de)coders, (de)modulation, (de)compression modules and to adapt said modules parameters to changing conditions, through the sharing of information about the source, network and channel states, and user requirements. For the controlling purpose, a signalling mechanism has been defined, as detailed in the following.

\section{SIDE INFORMATION EXCHANGED IN THE SYSTEM}

The information that is taken into account by the system for optimization is source significance information (SSI), that is information related to video content and representing the sensitivity of the source bitstream to channel errors; channel state information (CSI); decoder reliability information (DRI), that is soft values output by the channel decoder; source a-priori information (SRI), for example statistical information on the source, such as the a-priori knowledge on the prevalence of bits equal to 0 or 1 in the bitstream; source a-posteriori information (SAI), that is information only available after source decoding; network state information (NSI), represented, for instance, by packet loss rate and delay; and finally, the video quality measure, output from the source decoder and used as feedback information for system optimization.

When considering real systems, this control information needs to be transferred through the network and systems layers, in a timely and bandwidth efficient manner. Different mechanisms identified by the authors, which could allow information exchange transparently for the network layers (which we call the Network Transparency concept), are summarized in Table 1, together with the related overhead. Besides, one should not forget that several transport protocols exist, each of which can carry the payload and also some control information, for example datagram congestion control protocol (DCCP), UDP, UDP-Lite, and protocols at the transport layer.

Additional information is requested by the system for the setup phase, where information on available options is exchanged, the session is negotiated, and default parameters are set.

\section{JSCC/D CONTROLLERS}

The system controllers are represented by two distinct units, namely the physical layer (PHY) controller and the application layer (APP) controller. The latter collects information from the network (NSI: packet loss rate, delay, and delay jitter) and from the source (e.g., rate-distortion curves for the sequence under transmission, SSI) and has availability of reduced channel state information and of the quality metric of the previously decoded frame (or group of frames). According to this information, it produces controls for the source encoder block and for the network.

The physical layer controller's task is to provide controls to the physical layer blocks, that is the channel encoder, modulator, and interleaver, for example by performing UEP on the video data.

Adaptation to the source content, in terms of selective protection of semantically different portions of video data (UEP), may be performed at two different levels, either at the application layer (we call this content UEP, since content information is directly available there), or at the physical layer.

The application controller enables the manage- 


\begin{tabular}{lll} 
Control signal & Suitable Mechanism & Results \\
\hline SSI & IPv6 hop-by-hop or solutions as in [9] & $\begin{array}{l}\text { Overhead of few kb/s (depending on the source coding rate); high synchro- } \\
\text { nization with the video data. }\end{array}$ \\
\hline CSI & ICMPv6 & $\begin{array}{l}\text { Overhead of less than } 10 \mathrm{~KB} / \mathrm{s} \text { for CSI update frequencies up to } 10 \mathrm{~ms} \text {; } \\
\text { slight synchronization with the video data. }\end{array}$ \\
\hline NSI & ICMPv6 & Low overhead with suitable frequency of 100 ms (less than $1 \mathrm{Kbyte} / \mathrm{sec}$ ). \\
\hline DRI/SAI & IPv6 packets & $\begin{array}{l}\text { Very high bandwidth consuming (even higher than the video data flow of a } \\
\text { fixed multiplying factor). Probably better to send these control signals only } \\
\text { when the wireless receiver is also the data traffic destination. }\end{array}$ \\
\hline SRI & IPv6 hop-by-hop or destination & $\begin{array}{l}\text { Overhead of few Kbytes/sec (depending on the source coding rate) and } \\
\text { high synchronization with the video data. }\end{array}$
\end{tabular}

Table 1. Control and signal information transmission mechanisms and overheads.

ment of content adaptation at the application level, in which case one has direct access to the SSI, but only to reduced information on the channel status. When a standard radio access system is considered, performing UEP at the application layer allows standard compatibility. The physical controller manages content adaptation at the physical layer; in this case the SSI should transit through the network from the source to the channel, but quicker update based on detailed CSI can be done. In both cases, relevant information needs to be transmitted over the network and wireless channel to synchronize UEP at the receiver.

A more detailed description of the controllers is provided in the following.

\section{ApPlication LAYER CONTROLLER}

The application controller is considered as a finite-state machine, whether with a limited number of states for non-scalable video codecs, in which case state transitions are defined on the basis of pre-defined thresholds with respect to history and feedback information (video quality, NSI, reduced CSI), or with a non a-priori limited number of states for scalable video codecs, in which case the transitions are driven by fuzzy logic.

At the beginning of each iteration cycle of the duration, for instance, of one second (corresponding roughly to one or two groups of pictures, GOP), the controller decides the next operating state, defined by a set of configuration parameters for the different blocks of the chain, as illustrated in Fig. 2 for source encoder parameters.

To respect realism, the feedback information generated at the receiver side is relevant to the previous cycle, and is composed of:

- Video quality, either as a quality index without reference to the original sequence (similar to [10]), or with reduced reference. For easier evaluation of the results by the image and video coding community, the peak signal-to-noise ratio (PSNR) and other classical quality metrics (e.g., based on structural distortion [11]) are also used.

- Reduced CSI: average signal-to-noise ratio (SNR) in one controller step, channel coherence time.

- NSI: the number of lost packets, average jitter, average round trip time (RTT).
The main configuration parameters set by the application layer (APP) JSCC/D controller and modifiable at each simulation step are the video encoder frame rate, quantization parameters, GOP size, and the average channel code rate, as a consequence of the choice of the source encoding parameters and the knowledge of the available bandwidth. If UEP is applied at the application level, code rates $R_{c, i}$, where the index $i$ is related to the $i$ th sensitivity class, are also determined by the APP controller.

Given the bit-rate of the chosen state, the code rate available for signal protection is evaluated considering the total $R_{\max }=R_{s} / R_{c}$ constraint, where $R_{s}$ is the average source coding rate, including also the overhead due to the introduced network headers, and $R_{c}$ is the target average protection rate. The $R_{c}$ target information is used either for embedded unequal error protection at the application level as in [8], or provided directly to the physical layer controller. If physical layer UEP is adopted, given the available total coded bit rate $\left(R_{\max }\right)$, the average channel coding rate $\left(R_{c}\right)$ is derived by the application JSCC/D controller and proposed to the PHY controller.

The knowledge of the bit-rate is of course approximated and based on rate/source parameter models developed by the authors, or average values evaluated in previous controller steps.

The APP-controller follows slightly different rules according to the video coding standard considered. A different implementation has thus been provided for MPEG-4 video, H.264/AVC, and the fine granularity scalable version of the H.264 codec (SVC).

Example of APP Controller Behavior for MPEG-4 Video - In the case of MPEG-4 video, the controller considers frame rates of 30,15 , and $7.5 \mathrm{fps}$; spatial resolutions of QCIF, CIF; MPEG4 quantization parameters (frame I, frame P) equal to $(8,10)$ or $(14,16)$; and GOP length assuming values of 8,15 , and 30 . Furthermore, some constraints on these values must be satisfied, thus the number of the controller states is reduced.

As an example, for the first and second scenarios described in the following, five different states have been chosen for the application layer JSCC/D controller, characterized by different 
sets of values for the above mentioned parameters. State 1 corresponds to the lowest source data rate (lowest video quality) and highest robustness, whereas state 5 corresponds to the highest source data rate (highest video quality) and lowest robustness. Thus, decreasing the state number means increasing the robustness of transmission at the cost of a loss in the error free received video quality. Figure 2 depicts the finite state machine describing the APP-JSCC/D controller with the allowed transitions among states.

More precisely, the number of the possible sets of parameters is seven, since state 3 and state 5 have two different options for the GOP length. The choice of the GOP length is made according to channel conditions: for average channel $E_{b} / N_{0}$ (where $E_{b}$ is the energy per coded bit and $N_{0}$ is the one-sided thermal noise power spectral density) below a prefixed threshold, the lower GOP length is chosen, whereas for higher values of $E_{b} / N_{0}$ the higher GOP length is preferred. The different sets of parameters are reported in the figure, in terms of quantization parameters for I and $\mathrm{P}$ frames $(\mathrm{qI}, \mathrm{qP})$, frame rate $(\mathrm{Fr})$, and group of video objects (GOV) length (in our case a GOV is equivalent to a GOP, and the GOV length is equivalent to the $\mathrm{I}$ frames refresh rate). Basically, the adaptive algorithm which has been tested takes into account the trend of the video quality feedback from the source decoder, by suggesting a switch toward a more robust state when a negative trend is detected and the quality metric is below a threshold. When there is network congestion, indicated by a high value for the packet loss rate (PLR) feedback in the NSI, the controller sets immediately the state to the first, characterized by the lowest source bit rate, in order to reduce as much as possible the amount of data that have to flow through the IPv6 network. The hysteresis concept is used in order to avoid excessive oscillation between two states.

Fuzzy Logic Based APP Controller for SVC Video - Additionally, an alternative application controller for the H.264/SVC scalable video stream is considered. To fully exploit the scalable video stream, a large amount of states would be required, in order to allow fine granular adjustments to the video stream, thus a different approach using fuzzy logic has been used. The fuzzy controller sets the data rate and frame rate at the video sender by truncating the pre-encoded scalable video stream during the transmission. As input parameters it uses real-time transport control protocol (RTCP) statistics, previous control information, and channel capacity as additional cross-layer information from the physical layer. The control logic is based on several fuzzy logic rules that model the different states of the transmission system. Fuzzy combining is used to collect results from different rules and to provide output values for the data-rate and frame rate. The target is to maintain the highest possible data-rate for the video stream and react quickly on channel state degradation and recovery.

The controlling logic of the proposed controller contains 54 rules that can be summarized using the following aims:

- Try to increase the data rate if transmission conditions are good.

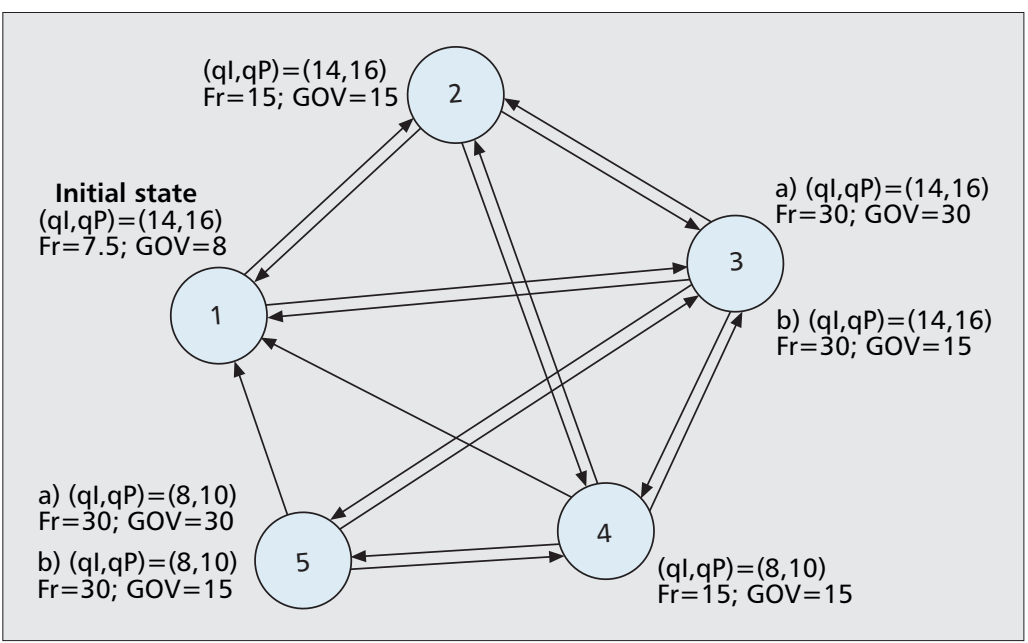

Figure 2. A graphical representation of the APP-JSCC as a finite state machine with five states. Example state sets for MPEG-4 video.

- If transmission conditions collapse, the data rate has to decrease rapidly.

- Try to find a stable state for the data rate and frame rate utilizing the feedback information.

- Try to find the maximum data rate of the system available and utilize it efficiently.

The main target of the controlling algorithm is to maintain the highest possible data-rate and perceived video quality for the video stream and at the same time utilize the available transmission capacity as efficiently as possible.

Adaptation is done by dropping the layers that do not fit into the estimated channel throughput. The last SNR layer to be included into the adapted bitstream is truncated to meet the bit budget requirement if it does not fit in it.

\section{Physical Layer Controller}

The physical layer controller's task is to decide, similar to [7], on the channel coding rate for each different sensitivity source layer, with the goal of minimizing the total distortion $D_{S+C}$ with the constraint of the average channel coding rate $R_{c}$, provided by the application controller. The "class conditional distortion curves," that is the curves representing the distortion (mean square error, MSE) vs. bit error rate (BER) for each class, when the remaining classes are errorless, have been modeled. The curves provide the sensitivity information on the source (SSI) needed for the UEP algorithm optimization. The model parameters also take into account the activity of the video sequence.

In particular, in the case of MPEG-4 video, the data partitioning tool has been exploited, separating I packets in a first class related to DC DCT coefficients and a second class related to AC DCT coefficients, whereas $P$ packets are partitioned into a first class containing motion information and a second class containing texture information. UEP is performed on the bitstream according to the sensitivity of the four classes to channel errors, modeled through the curves described above. The video content is thus critical information, here exploited in terms of SSI and of video activity (influencing the sensitivity model).

We propose in fact to choose channel coding 


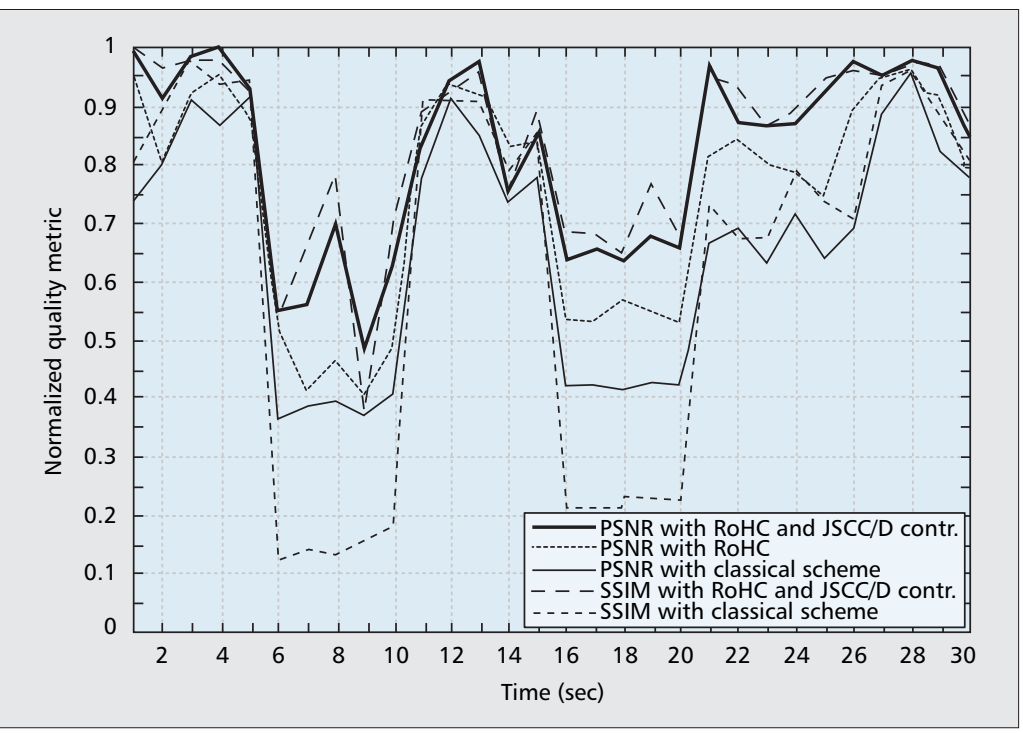

Figure 3. Received video quality versus time with the JSCC adapted and nonadapted systems. Foreman sequence in CIF format. MPEG-4 source encoding.

rates for each different sensitivity partition on the basis of the produced rate-distortion. Considered a source represented by a bitstream that may be separated into layers or partitions of different significance, each partition may be protected with a different channel coding rate, according to its sensitivity to channel errors. Each partition, with bit-rate $R_{s, i}$, is protected with an error correcting code with code rate $R_{c, i}$, such that the total source and channel coding rate is

$$
R_{s+c} \leq \sum_{i=1}^{N} \frac{R_{s, i}}{R_{c, i}},
$$

where $N$ is the number of partitions considered.

Furthermore, the controller sets the parameters for bit-loading in multicarrier modulation and interleaver characteristics, and performs a trade-off with receiver complexity.

\section{HeAder Compression}

In order to limit the potentially dramatic impact of errors on RTP/UDP-Lite/IPv6 headers over the wireless link, a header compression module based on IETF robust header compression (RoHC) recommendations has been introduced in the transmission chain. Located near the wireless part immediately after IPv6 wired network and mobility modeling modules, this module provides the compressed network header concatenated with the unchanged video payload to the Data link module. In practice, the uncompressed RTP/UDP-Lite/IPv6 header of size 60 bytes can be reduced in average to a value of 5 bytes only. This header size reduction implies a large increase in terms of robustness to errors, as shown in the results section, since more protection can be provided to headers if their size is reduced.

\section{The Demonstration Platform}

In order to provide a realistic performance evaluation of the proposed approach, all the involved system layer blocks have been realistically implemented. Namely:
- Application layer controller, also including an optional content level UEP block (using rate compatible punctured convolutional, RCPC, codes).

- Source encoder/decoder (three possible codecs: MPEG-4, H.264/AVC, and Scalable Video Coding in H.264/AVC Annex G), where soft-input source decoding is also allowed for H.264/AVC.

- Cipher/decipher unit.

- Real-time transport protocol (RTP) header insertion/removal.

- Transport protocol header (e.g., UDP-Lite, UDP, or DCCP) insertion/removal.

- IPv6 header insertion/removal.

- IPv6 mobility modeling.

- IPv6 network simulation.

- Robust Header Compression (RoHC)

- DLL header insertion/removal.

- Radio Link, including

- Physical layer controller.

- Channel encoder/decoder (convolutional, RCPC, low density parity check (LDPC) codes with soft and iterative decoding allowed).

- Interleaver.

- Modulator (OFDM, TCM, TTCM, STTC), with soft and iterative demodulation allowed.

- Simulated channel (e.g., AWGN, Rayleigh fading, shadowing, frequency selective channels).

\section{ENVISAGED SCENARIOS}

In order to emphasize the interest of the end-toend optimization strategy developed for the transmission of multimedia data over an IP wireless link, different scenarios have been identified in which this optimization would be interesting for the enduser or the provider. A first scenario consists of applications such as video conference on the move. In this case the main characteristics are conversational mode, UMTS channel (or 4G), multicast, a mobile phone as the terminal device, no strict cost limitations, confidentiality, and multi-user systems.

In the case of a stationary video conference (e.g., from a cafe) considered in the second scenario, the same parameters as in the first scenario are used, but a WLAN channel is taken into account instead of UMTS. If scalable video coding (SVC) is used, the image size can be adapted in the network according to the capabilities of the terminal. Further scenarios have been identified, such as video on demand and learning applications, video calls, stationary or on the move, and pushed video information (e.g., live news).

\section{Simulation Results}

Figure 3 shows a performance comparison among examples of video transmissions adopting different strategies, from the "classical" scheme to the proposed joint-adaptive techniques at the application and physical layer. The comparison is made in terms of subsequent video quality values, each obtained through the average over $1 \mathrm{~s}$ of five simulations run with different noise seeds. The values obtained have been normalized (by setting the maximum value to 1 ), in order to allow a direct comparison between different quality indexes: 
PSNR and structure similarity metric [11]. The scenario considered is the second scenario described above, with a WLAN supporting, at the radio link level, a coded bitrate of $12 \mathrm{Mb} / \mathrm{s}$. The video stream is coded according to the MPEG-4 standard and is supposed to be multiplexed with other real-time transmissions so that it occupies only an average portion of the available bandwidth, corresponding to a coded bitrate of $1 \mathrm{Mb} / \mathrm{s}$. The Foreman sequence in CIF resolution has been selected according to the considered scenario. The APP controller states, representing the possible quantization parameters, frame rate, and GOP rate, are those represented in Fig. 2. The channel code is an IRA-LDPCC with a "mother" code rate of $(3500,10500)$, properly punctured and shortened in order to obtain different code rates. The resulting codewords are 4200 bits long. The code rate is fixed for the non adapted system (meaning that an equal error protection, EEP, policy is adopted) and its value is determined according to the constraint on the maximum allowed coded bitrate (i.e., $1 \mathrm{Mb} / \mathrm{s})$. In contrast, in the adapted case the code rate can change according to the SSI in order to perform UEP. In the reference transmission scheme, the modulation is orthogonal frequency division multiplexing (OFDM) with 48 carriers for data transmission and a frame duration of $4 \mu \mathrm{s}$, whereas the PHY-JSCC also applies margin adaptive bit-loading. The simulated channel is obtained according to the ETSI standard channel $\mathrm{A}$, and it also takes into account a log-normal flat fading component with channel coherence time of $5 \mathrm{~s}$, to consider the fading effects due to large obstacles. The figure is related to $E_{b} / N_{0}=11.2 \mathrm{~dB}$, where $E_{b}$ is the energy per coded bit.

From the curves in Fig. 3 the gain achieved with JSCC/D controllers and with RoHC is evident. In the reference system, no RoHC is adopted, nor adapting algorithms. When RoHC is enabled, it allows a reduction in the overhead due to the header, and a channel code with a lower code rate can be applied, determining an average PSNR improvement of $2.73 \mathrm{~dB}$. If both RoHC and JSCC/D controllers are enabled the improvement is particularly evident, with any considered metric. In particular, we observed an average gain of $5 \mathrm{~dB}$ in terms of PSNR in the conditions under analysis.

As a different example, results obtained with the H.264/AVC codec and the Akiyo video sequence in QCIF $15 \mathrm{~Hz}$ format are reported in Fig. 4 . The adaptation is there done only by the APP controller for a source and application protection global rate of $128 \mathrm{~kb} / \mathrm{s}$, transmitted over a slow fading channel with coherence time of 10s. In the adapted case, a joint optimization of the source compression and protection by means of UEP with RCPC codes at the application layer is performed, to be compared to a fixed compression of $64 \mathrm{~kb} / \mathrm{s}$ and EEP protection of rate of $1 / 2$. In both cases, $\mathrm{RoHC}$ compression is used, leading to an average gain of $2.8 \mathrm{~dB}$ in terms of PSNR in the adapted case. This gain corresponds to a better adaptation to channel variations thanks to the access to reduced CSI, in particular when the channel is highly impaired. Although the adapted stream can sometimes perform slightly worse than the nonadapted stream, mostly when the APP controller leads to compress more (by resorting to default ini-

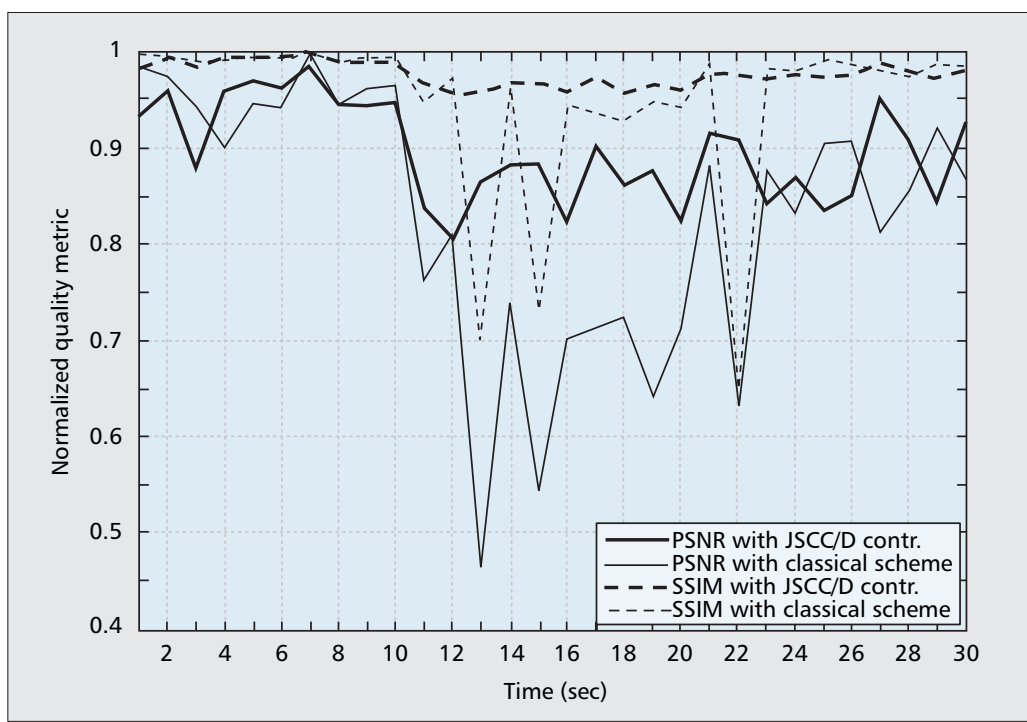

Figure 4. Received video quality vs. time with the JSCC adapted and nonadapted systems. Akiyo sequence in QCIF format. H.264/AVC source encoding.

tial settings or following a bad return information), the considered cross-layer approach is obviously much more robust than the reference approach.

Visual results, in accordance with average visual impact, are reported in Fig. 5, where as an example the received frame no. 435 for the CIF Foreman sequence and no. 262 for the QCIF Akiyo sequence are reported, both for the adapted and the non-adapted case. The original frame is reported for comparison.

\section{CONCLUSIONS}

A global approach for realistic network-aware joint source and channel system optimization has been outlined in the article. A remarkable gain is obtained with the developed demonstrator when the adaptation options are activated, although more realistic assumptions than those commonly considered in the literature, in terms of redundancy due to side information transmission, are made. The results could thus be seen as a proof of the real applicability of joint source and channel coding techniques and of the gain achievable through cross-layer design.

\section{ACKNOWLEDGMENT}

This work has been partially supported by the European Commission in the framework of the "PHOENIX" IST project under contract FP6-2002IST-1-001812. All the "PHOENIX" IST project consortium partners, contributing to the development and implementation of the described system, are gratefully acknowledged. We would like to thank in particular Janne Vehkaperä (VTT) for his work on SVC, Ing. Gianmarco Panza (CEFRIEL) for his contribution in Table 1, and Ph.D. student Cyril Bergeron (THALES Communications) for his help in running the H.264/AVC simulations.

\section{REFERENCES}

[1] J. L. Massey, "Joint Source and Channel Coding," Commun. Systems and Random Process Theory, NATO Advanced Studies Institutes Series E25, J.K. Skwirzynski editor, Sijthoff \& Noordhoff, Alphen aan den Rijn, The Netherlands, 1978, pp. 279-93. 
[a]

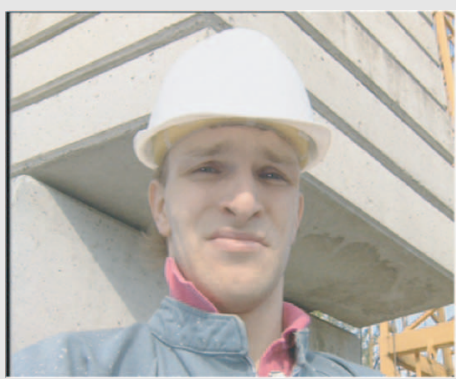

[d]

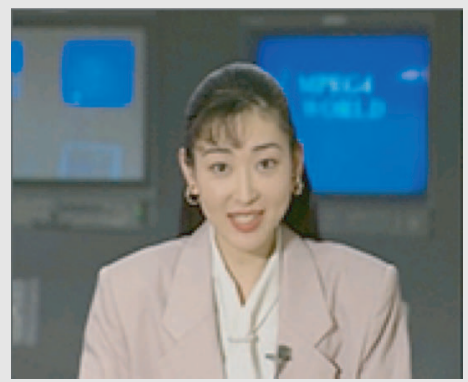

[b]

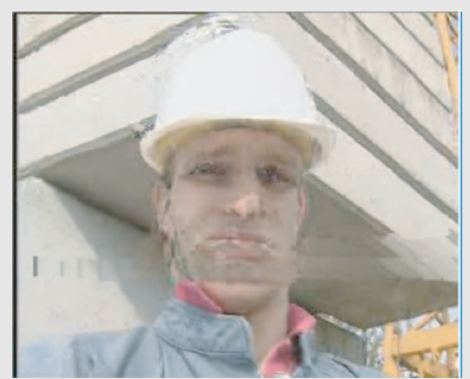

[e]

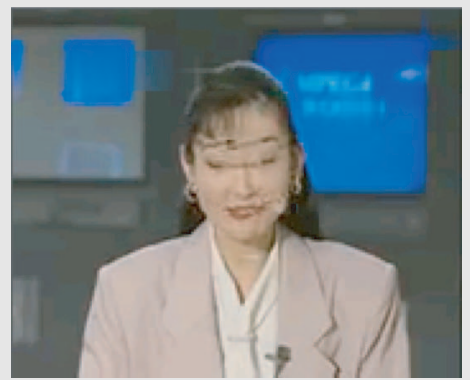

[c]

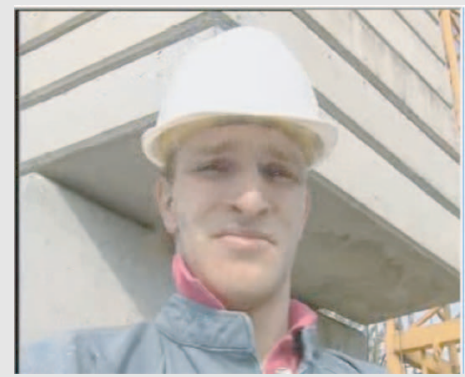

[f]

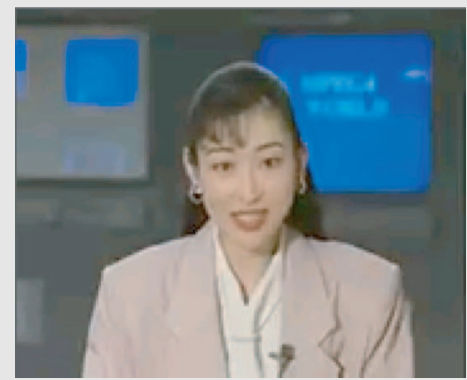

Figure 5. Visual results: a) Frame no. 435 of the Foreman sequence - original; b) Frame no. 435 of the Foreman sequence - MPEG-4 - no RoHC, no JSCC, c) Frame no. 435 of the Foreman sequence - MPEG-4 - RoHC, JSCC; d) Frame no. 262 of the Akiyo sequence - original; e) Frame no. 262 of the Akiyo sequence - H.264/AVC - EEP; and f) Frame no. 262 of the Akiyo sequence - H.264/AVC UEP at application layer.

[2] C. E. Shannon, "A Mathematical Theory of Communication," Bell System Technical J., vol. 27, July-Oct. 1948 pp. 379-423, 623-56.

[3] J. Hagenauer and T. Stockhammer, "Channel Coding and Transmission Aspects for Wireless Multimedia," Proc. IEEE, vol. 87, no. 10, Oct. 1999, pp.1764-77.

[4] J. Hagenauer, N. Seshadri, and C. E. Sundberg, "The Performance of Rate-Compatible Punctured Convolutional codes for Digital Mobile Radio," IEEE Trans. Commun., vol. 38, no. 7, July 1990, pp. 966-80.

[5] J. Modestino, D. Daut, "Combined Source-Channel Coding of Images," IEEE Trans. Commun., Nov. 1979, vol. 27, no. 11, pp. 1644-59.

[6] M. van der Schaar et al., "Adaptive Cross-Layer Protection Strategies for Robust Scalable Video Transmission Over 802.11 WLANs," IEEE JSAC, vol. 21, Dec. 2003 pp. 1752-63.

[7] M. G. Martini and M. Chiani, "Rate-Distortion models for Unequal Error Protection for Wireless Video Transmission," IEEE VTC 2004 Conf., Milan, Italy, May 2004.

[8] C. Lamy-Bergot, N. Chautru, and C. Bergeron, "Unequal Error Protection for H.263 + Bitstreams Over a Wireless IP Network," IEEE Int'l. Conf. Acoustics, Speech and Sig. Proc. (ICASSP'06), Toulouse, France, May 2006.

[9] M. G. Martini and M. Chiani, "Proportional Unequal Error Protection for MPEG-4 Video Transmission," Proc. IEEE ICC 2001, Helsinki, June 2001.

[10] Z. Wang, H. R. Sheikh, and Alan C. Bovik, "No-Reference Perceptual Quality Assessment of JPEG Compressed Images," Proc. IEEE Int'l. Conf. Image Proc., Rochester, New York, Sept. 22-25, 2002, pp. 477-80.

[11] Z.Wang, L. Lu, and A. C. Bovik, "Video Quality Assessment Based on Structural Distortion Measurement," Signal Processing: Image Communication, vol. 19, no. 2, Feb. 2004, pp. 121-32.

\section{BIOGRAPHIES}

MARIA G. MARTINI (maria.martini@cnit.it) is a postdoctoral researcher with CNIT-DEIS, University of Bologna, Italy. Her research interests are mainly in video coding, channel coding, joint source and channel coding, error-resilient video transmission, cross-layer design for wireless multimedia, decision theory, frame synchronization. She is the author of several scientific papers and patents in the field of wireless video transmission. She holds a Laurea (M.Sc.) degree in electronic engineering (magna cum laude) from the Uni- versity of Perugia (1998) and a Ph.D. in electronics and computer science from the University of Bologna (2002)

Matteo Mazzotti (mmazzotti@deis.unibo.it) received the degree in telecommunication engineering (with the highest honors) in July 2002 from the University of Bologna. Now he is working as a Ph.D. student in the Dipartimento di Elettronica, Informatica e Sistemistica in the University of Bologna. His main research interests cover multimedia communications, joint source and channel coding, and wireless communication systems.

CATHERINE LAMY-BERGOT (catherine.lamy@fr.thalesgroup.com received in 1996 both the electrical engineering and M.Sc. (DEA) degrees from the Ecole Nationale Supérieure des Télécommunications, Paris, France, and the Ph.D. degree in 2000. She is currently a senior scientist in digital communications with THALES Communications, Colombes, France, where she leads the IST PHOENIX project. Her fields of interest include iterative decoding techniques, high efficiency modulations, error correction codes, unequal error protection, soft-input/soft-output decoding, joint source and channel coding techniques, robust header compression and cross-layer optimisation.

JYRKı HUUSKo (Jyrki.Huusko@vtt.fi) received his degree in theoretical physics with minor subjects in information technology and mathematics from the University of Oulu. He is working at the VTT Technical Research Centre of Finland as a research team leader on the Seamless Networking team. His current research topics include future autonomic networks and services, transport protocols and multimedia delivery optimization, cross-layer communication design in heterogeneous wireless and mobile networks, cross-layer communication aided network mobility and multi-access.

PETER AMOn (p.amon@siemens.com) received his diploma in electrical engineering from the University of Erlangen, Germany in 2001, where he specialized in communications and signal processing. In 2001 he joined Siemens Corporate Technology, Information and Communications, where he is currently working as a senior research scientist in the Networks and Multimedia Communications department. His research field encompasses video coding, video transmission, error resilience and joint source channel coding. In that area, he has published several conference papers and is also actively contributing to the standardization bodies ITU-T and ISO/IEC MPEG. 